medication. For example, the 1994 VIDAL (the French equivalent of the British National Formulary) gives an advisory maximum daily dose of the oral antipsychotic fluphenazine of $800 \mathrm{mg}$, and an average daily dose of $25-300 \mathrm{mg}$, compared to a $20 \mathrm{mg}$ maximum daily dose in the BNF (a $4000 \%$ difference). Further research into the relative merits of these divergent treatment approaches is clearly needed.

Thompson, C. (1994) The use of high-dose antipsychotic medication. British Journal of Psychiatry, 164, 448-459.

VAN OS, J., GAldos, P., LeWIS, G., et al (1993) Schizophrenia sans frontières: concepts of schizophrenia among French and British psychiatrists. British Medical Journal, 307, 489-492.

S. Dollfus

Centre Hospitalier et Universitaire

Côte de Nacre, Caen 14000, France

Institute of Psychiatry

J. VAN OS

De Crespigny Park

London SE5 8AF

Centre Hospitalier du Rouvray

Sotteville les Rouen 76301, France

M. Petit

\section{Lithium in steroid-induced depression}

SIR: In their report of two cases of successful lithium treatment of mood disorders associated with corticosteroid treatment, Terao et al (BJP, January 1994, 164, 109-111) conclude that the effectiveness of lithium in these cases is a manifestation of its activity as a serotonergic antidepressant. This interpretation is tenable, but another interpretation must also be considered.

Mixed mood disturbances (referred to as agitated depression, dysphoric mania, or mixed manicdepressive states) can closely resemble other depressive states (McElroy et al, 1992). If these two patients were suffering from mixed mood disturbances, then their positive responses to lithium would be seen as responses to a mood-stabiliser. Note that each of these patients had anxiety and irritability as prominent symptoms.

The authors' interpretation would predict a positive response in these cases to serotonergic antidepressants. Although specific serotonergic drugs have not been systematically studied in these patients, reports on responses to older non-specific antidepressants (each of which has some serotonergic activity) have been very negative (Hall et al, 1978). Authors who had previously reported positive results of lithium treatment for steroid-induced mood disturbances had noted the high incidence of manic and mixed manic-depressive states in these patients as a reason for trying lithium (Siegal, 1978; Falk et al, 1979).

By the same token, an interpretation based on mood stabilisation would predict a positive response to other mood stabilisers, such as carbamazepine or valproate. Consider the following case. A 41-year-old female was being treated for Crohn's Disease of long duration with prednisone in doses as high as $60 \mathrm{mg}$ per day. All other treatments had failed. She found that irritability, racing thoughts, emotional lability and dysphoria appeared whenever high-dose prednisone was used. I rejected lithium because it irritates the gastrointestinal tract. Carbamazepine, in a dose of $800 \mathrm{mg}$ per day, brought about complete cessation of her mood disturbance even at the highest prednisone doses.

Steroids are frequently used in high doses. More research on the treatment of steroid-induced mood disturbances would be very useful.

Falk, W.E., Mahnke, M.W. \& Poskanzer, D.C. (1979) Lithium prophylaxis of corticotrophin-induced psychosis. Journal of the American Medical Association, 241, 1011-1012.

Hall, R.C.W., Popkin, M.K. \& KirkPatrick, B. (1978) Tricyclic exacerbation of steroid psychosis. Journal of Nervous and Mental Disease, 166, 738-742.

McElroy, S.L., KeCK, P.E., Pope, H.G., et al (1992) Clinical and research implications of the diagnosis of dysphoric or mixed mania or hypomania. American Journal of Psychiatry, 149, 1633-1644.

SiEGAL, F.P. (1978) Lithium for steroid-induced psychosis. New England Journal of Medicine, 299, 155-156.

D.J. LYNN

St. Francis Medical Center

400-45th Street

Pittsburgh, PA 15201-1198

\section{Language and psychiatry}

SIR: While welcoming Thomas \& Fraser's review of recent developments in linguistics $(B J P$, November $1994,165,585-592)$, I am surprised that no mention of any psychoanalytic works was made, as psychoanalysis is above all a textual analysis. The French psychoanalyst Jacques Lacan said that "psychoanalysis has only one medium: the patient's speech", thus rehabilitating speech and language as the basis of Freudian analysis (Lacan, 1953).

Freud's command to his first patients (the hysterics for whom speech was so problematic) was to speak. It was through this speech that the unconscious text emerged. His example of the fort-da game (Freud, 1920) shows how the child uses language in order to cope with the mother's absence 